\title{
Vômitos em traumatismo cranioencefálico leve na infância
}

\author{
Carlos Umberto Pereira', Alyne Andrade Lima² \\ Universidade Federal de Sergipe (UFS), Serviço de Neurocirurgia do Hospital de Urgência de Sergipe Governador João \\ Alves Filho (HUSE), Aracaju, SE, Brasil.
}

\section{RESUMO}

Objetivo: O presente trabalho visa identificar o significado do vômito em crianças com traumatismo cranioencefálico (TCE) leve. Métodos: O presente trabalho foi prospectivo e descritivo em 88 pacientes com idade abaixo de 16 anos, atendidos no setor de urgência do HUSE (Aracaju-Sergipe). Foram analisados idade, gênero, causas do trauma, quadro neurológico, exames complementares e conduta. Resultados: Foram analisados 88 pacientes. O gênero M 74/F 14 casos. A média das idades foi de 5,5 anos. As causas do TCE foram: queda acidental (62 casos), seguida de acidente de trânsito (10 casos), queda de bicicleta (9 casos), maus-tratos (2 casos) e desconhecido (5 casos). A média de episódios de vômitos foi de dois. Outras queixas foram cefaleia e sonolência. Raios $x$ simples de crânio realizados em 84 casos demonstraram fratura linear em dois e afundamento craniano em um. TC realizada em 88 pacientes revelou hematoma extradural (1), hematoma subdural (1), hemorragia subaracnóidea (1) e contusão temporal (1). Tratamento cirúrgico foi instituído em dois casos. Não houve relação entre o número de episódios de vômitos e achados de imagem. Achados anormais do exame de raios $x$ simples associados com vômitos e sonolência foram preditores de lesão cerebral. Não houve óbito. Conclusão: O conhecimento do significado de vômito em TCE leve na infância é imprescindível para evitar a realização desnecessária de exames de imagem que acarretam riscos biológicos e custos adicionais. Não encontramos relação entre vômitos e lesão cerebral em crianças com TCE leve. Ausência de vômitos em crianças com TCE leve não exclui ausência de lesão cerebral.

\section{PALAVRAS-CHAVE}

Vômitos, traumatismos craniocerebrais, criança, prognóstico.

\section{ABSTRACT}

Vomiting in mild traumatic brain injury during childhood

Objective: This present study aims to show the real importance of vomiting post-traumatic in children who had mild head trauma. Methods: A prospective and descriptive study off 88 patients from birth to 16 years with minor head injury who were presented to as emergency service in the HUSE (AracajuSergipe). Characteristics studied were age, sex, mechanisms of injury, site of impact, neurological on arrival, exams and treatment. Results: Eighty eight patients presented vomiting post traumatic as the most important symptom and this was the study group. Gender male74/female 14 cases. The average age was 5,5 years. Most injuries were caused by a fall (63 cases). The mechanism of injury was a fall in 62, involvement in a road traffic accident in 10, bicycle crash in 9, abuse in 2 and unknown in 5 . The average of episodes of vomiting were two. Other complaints were headache and somnolence. A simple skull $x$-ray performed in 84 cases demonstrated a linear fracture in two and compound fracture of skull in one. CT performed in 88 patients revealed one extradural hematoma, one subdural hematoma, one subarachnoid hemorrhage and one temporal contusion. Surgical treatment was instituted in two cases. There was no finds compared between the number of episodes of vomiting and imaging findings. Abnormal findings on simple $x$-ray associated with vomiting and somnolence were predictors of brain damage. There was no death. Conclusion: The knowledge of the significance of vomiting in mild head trauma in children is essential to avoid performing unnecessary imaging tests that carries biological risks and additional costs. There was no compared between brain injury and vomiting in children with mild head injuries. No vomiting in children with mild head trauma doesn't exclude the absence of brain damage.

\section{KEYWORDS}

Vomiting, craniocerebral trauma, child, prognosis.

1 Professor doutor do Departamento de Medicina da Universidade Federal de Sergipe (UFS), médico do Serviço de Neurocirurgia do Hospital de Urgência de Sergipe Governador João Alves Filho (HUSE), Aracaju, SE, Brasil.

2 Acadêmica de Medicina da UFS, Aracaju, SE, Brasil. 


\section{Introdução}

Entende-se por traumatismo cranioencefálico (TCE) qualquer agressão de origem traumática que provoque lesão anatômica ou comprometimento funcional do couro cabeludo, crânio, meninges, encéfalo ou seus vasos. O TCE é responsável por $75 \%$ a 97\% dos óbitos devidos a traumatismo em crianças, tornando-se, assim, um desafio da saúde pública na infância e adolescência. ${ }^{1} \mathrm{~A}$ incidência de casos nos Estados Unidos é de 600.000 atendimentos por ano em departamentos de emergência, 95.000 internações e 7.000 óbitos. $^{2}$

Em sua maioria, os casos de TCE em unidades de emergência são leves, porém podem causar lesões graves. ${ }^{2}$ As principais causas de TCE leve na infância são motivadas por quedas de própria altura, seguidas por quedas de bicicleta e acidentes de trânsito. ${ }^{3-5}$ No TCE leve, apenas um número reduzido de casos requer exames de imagens. ${ }^{6}$ Os principais critérios de indicação para realização de tomografia computadorizada (TC) são: escore na escala de coma de Glasgow (ECG) menor ou igual a 12 , perda de consciência e vômito. ${ }^{6}$ Vômito pós-TCE (VPTCE) tem sido associado com fratura craniana e hematoma intracraniano. ${ }^{7,8}$ Porém, ainda não está claro se o vômito é um fator de risco independente para lesões intracranianas. ${ }^{9}$

Alguns autores sugerem, em casos de vômitos e cefaleia pós-TCE, a realização de TC e internação para observação, por provável lesão cerebral. ${ }^{710}$ Nee et al. ${ }^{11}$ demonstraram que a presença de VPTCE aumenta em quatro vezes o risco de fratura de crânio em pacientes alertas, sem diferenças entre crianças e adultos. Outros autores defendem que o vômito como sintoma isolado não predispõe a riscos de lesão cerebral grave. ${ }^{4,5,12}$ Ainda se verificou que não havia diferenças quanto ao prognóstico entre pacientes que apresentavam ou não vômitos. ${ }^{12}$ Apesar de todas as controvérsias, é consenso entre os autores que o número de episódios de vômito não aumenta a predisposição à lesão intracraniana, assim um simples episódio não seria mais importante que vários. ${ }^{5,10,12}$

Os autores apresentam sua experiência com crianças, vítimas de traumatismo cranioencefálico leve, e discutem sobre a presença de vômitos e sua associação com o prognóstico do paciente. A presença de vômitos em pacientes com TCE leve em alguns serviços é um dos requisitos usados pelos médicos para indicação de TC. O estudo sobre a influência desse sinal no prognóstico e lesão nos pacientes é de fundamental importância, pois seleciona os principais casos que realmente necessitam do exame, reduzindo, assim, o uso de radiação em crianças e também os custos hospitalares.

\section{Pacientes e métodos}

O presente trabalho foi prospectivo e descritivo. Foram estudados 88 pacientes abaixo de 16 anos de idade, vítimas de traumatismo cranioencefálico leve, atendidos na Unidade de Emergência do Hospital de Urgências de Sergipe (HUSE), apresentando vômitos e cefaleia.

Em nosso estudo, consideramos com TCE leve crianças que se apresentavam com história de diminuição da consciência, amnésia ou desorientação e com escore na ECG entre 14 e 15.

Elas foram analisadas quanto a idade, gênero, causas do trauma, quadro clínico, exame neurológico, exames complementares, conduta e tratamento.

\section{Resultados}

Foram estudados 88 pacientes, sendo 74 do sexo masculino e 14 do sexo feminino. A média das idades foi de 5,5 anos. As principais causas foram: queda acidental/ altura, com 62 casos, seguida de acidentes de trânsito (10 casos), queda de bicicleta ( 9 casos), maus-tratos (2 casos) e desconhecido (5 casos). A média de episódios de vômito foi dois, com variação de 1 a 7 episódios.

$\mathrm{O}$ exame de raios $\mathrm{x}$ simples de crânio foi realizado em 84 casos, revelando fratura linear em dois casos e afundamento craniano em outro caso. A TC foi realizada em todos os pacientes e revelou hematoma extradural (1 caso), hematoma subdural (1 caso), hemorragia subaracnóidea (1 caso), contusão cerebral (1 caso) e afundamento craniano (1 caso). Dois pacientes foram submetidos à intervenção cirúrgica. Não houve óbito.

\section{Discussão}

De acordo com o Mild Trauma Brain Injury Committee of the Head Injury Interdisciplinary Special Interest Group of the American Congress of Rehabilitation Medicine, TCE leve é qualquer trauma ou disfunção cerebral que leve a, no mínimo, um desses sintomas: perda de consciência por 30 segundos ou menos, perda de memória imediatamente antes ou depois do acidente, que não exceda 24 horas, e alteração do estado mental com escore na ECG após 30 minutos de 13 a $15 .^{13}$

O sexo masculino foi o mais acometido, com $84 \%$ e $16 \%$ do sexo feminino. As principais causas de TCE leve na infância foram similares às demonstradas por outros autores, ${ }^{14,15}$ assim como foram idênticos os sintomas de TCE leve na infância: vômitos, sonolência e cefaleia. ${ }^{15}$ 
VPTCE são mais comuns em crianças por causa do comprometimento vestibular transitório e podem iniciar-se imediatamente ou horas após o trauma, ocorrendo em episódios únicos ou múltiplos. ${ }^{16} \mathrm{O}$ mecanismo exato dos VPTCE é controverso, mas é provável que as forças de impulso sejam mais importantes que o próprio impacto. ${ }^{11}$ Assim, enquanto fraturas seriam causadas por forças de contato, náuseas e vômitos seriam decorrentes do movimento do trauma. Provavelmente, uma força cinética grande para causar fratura de crânio também seria responsável pela movimentação do crânio e consequente presença de vômito, porém o contrário não ocorre. ${ }^{17}$ Portanto, a associação entre os dois sinais é frequente, mas não exata, sendo maior em adultos. ${ }^{18}$ Em nosso trabalho não foi observada relação entre o número de episódios de VPTCE e lesão intracraniana na infância, fato esse observado na literatura médica. ${ }^{11,19}$

A literatura médica demonstra a associação entre vômito, bradicardia, hipertensão arterial sistêmica e alterações pupilares durante hipertensão intracraniana ocasionada por TCE, sendo o vômito provavelmente devido a um edema, distorção ou isquemia do tronco cerebral. ${ }^{20}$ Vários autores têm relacionado VPTCE a fatores familiares como história de cefaleia e enjoos. ${ }^{21,22}$ Se a cefaleia for do tipo enxaqueca, a probabilidade de VPTCE está aumentada. ${ }^{5,23}$ Quando mais de um desses fatores está presente, a probabilidade de vômito é de $100 \% .{ }^{23}$

A literatura tem relacionado a presença de vômitos com fratura craniana. É importante lembrar que a associação entre fraturas cranianas e lesões intracranianas não é tão forte quanto em adultos. ${ }^{18}$ Estudos mostram que a ocorrência de VPTCE é um fator independente associado com maior incidência de fratura craniana em pacientes alertas. ${ }^{11}$ No presente trabalho não houve evidências concretas de tal associação, observando-se que o vômito não é um fator isolado de risco para pacientes vítimas de TCE leve.

A TC em TCE leve na infância tem indicação quando existem critérios clínicos associados a vômito, e não como sintoma isolado. Evita-se, dessa forma, que sejam realizados exames desnecessários, que em muitos casos necessitam de sedação, provocam danos biológicos e aumentam os gastos hospitalares..$^{24}$ Por outro lado, um bom entendimento sobre o significado do VPTCE também se faz necessário quanto à conduta adequada a ser seguida em relação ao paciente vítima do TCE, para que sejam evitadas lesões não diagnosticadas.

A literatura médica tem demonstrado a relação entre vômito, idade e local do trauma na criança. Existe maior suscetibilidade a VPTCE em crianças abaixo de 2 anos de idade, observando-se maior incidência quando o trauma ocorre em região occipital. ${ }^{12}$ É importante atentar para o possível envolvimento do transporte dos pacientes até o hospital como possível causa adicional dos VPTCE. Em nosso estudo, observou-se que crianças menores de 2 anos de idade estão mais predispostas a apresentar vômitos, assim como traumas na região occipital.

A presença de náuseas e vômitos, além de cefaleia intensa, diminuição transitória do nível de consciência, amnésia anterógrada e crises convulsivas, é uma das indicações para a realização da TC. ${ }^{25}$ A escolha do exame ideal para cada tipo de trauma é essencial para o diagnóstico de possíveis lesões. $\mathrm{O}$ vômito tem sido critério adotado por alguns estudos como o New Orleans Criteria (NOC) e o Canadian CT Head Rule (CCHR) para a realização de TC em TCE leve. ${ }^{6,19}$ O NOC apresenta o vômito, com dois ou mais episódios, como fator de alto risco para realização de TC; já o CCHR não especifica a quantidade de episódios de vômitos. ${ }^{19}$ Porém, a especificidade desse sintoma na predição de lesão intracraniana é baixa, especialmente em crianças abaixo de 3 anos de idade. ${ }^{26}$

Alguns autores relacionam a presença de VPTCE como fator de risco de lesão intracraniana em pacientes com nível de consciência preservado. ${ }^{27,28}$ Nosso trabalho está de acordo com outros autores para os quais episódio de vômito isolado pós-TCE não é fator de risco independente. ${ }^{4,12,21,22}$

A incidência de achados positivos em TC realizada em pacientes com TCE leve é abaixo de $10 \%$, e menos de $1 \%$ necessita de intervenção cirúrgica. ${ }^{29,30}$ Nosso estudo ratifica a literatura, com apenas $1,25 \%$ dos 1.160 casos de TCE leve necessitando de intervenção cirúrgica, confirmando o fato de que o TCE leve, apesar de ser o trauma mais comum, não é o mais perigoso. Porém, mesmo em menor percentual, os casos com necessidade de intervenção devem ser diagnosticados rapidamente, para que haja tratamento ideal, pois o tempo entre o diagnóstico e o tratamento é essencial para o prognóstico do paciente. A maioria das crianças com TCE leve, escore na ECG de 15 e exame neurológico normal pode ser liberada para o lar, mas com orientação para, em caso de piora, retornar ao hospital. ${ }^{31}$

\section{Conclusão}

No presente estudo não houve relação entre vômitos e lesão cerebral em crianças com TCE leve. Da mesma forma, não foi verificada relação entre o número de episódios de vômitos e os achados de imagem. A ausência de vômitos em crianças pós-TCE leve não exclui a ausência de lesão cerebral. Os preditores de lesão cerebral verificados em nosso trabalho foram achados anormais de radiografia de crânio associados a vômitos e sonolência. 


\section{Conflito de interesses}

Não houve conflito de interesses na realização deste trabalho.

\section{Referências}

1. Guerra SD, Jannuzzi MA, Moura AD. [Pediatric head injury]. J Pediatr (Rio J). 1999;75(Suppl 2):S279-93.

2. Schutzman SA, Greenes DS. Pediatric minor head trauma. Ann Emerg Med. 2001;37(1):65-74.

3. Jennett B. The problem of mild head injury. Practitioner. 1978;221(1321):77-82.

4. Leonidas JC, Ting W, Binkiewicz A, Vaz R, Scott RM, Pauker $S G$. Mild head trauma in children: When is a roentgenogram necessary. Pediatrics. 1982;69(2):139-43.

5. Brown FD, Brown J, Beattie TF. Why do children vomit after minor head injury? J Accid Emerg Med. 2000;17(4):268-71.

6. Livingston $\mathrm{DH}$, Lavery RF, Passannante MR, Skurnick $\mathrm{JH}$, Baker S, Fabian TC, et al. Emergency department discharge of patients with a negative cranial computed tomography scan after minimal head injury. Ann Surg. 2000;232(1):126-32.

7. Masters SJ, McClean PM, Arcarese JS, Brown RF, Campbell $J A$, Freed HA, et al. Skull x-ray examinations after head trauma. Recommendations by a multidisciplinary panel and validation study. N Engl J Med.1987;316(2):84-91.

8. Duus BR, Boesen T, Kruse KV, Nielsen KB. Prognostic signs in the evaluation of patients with minor head injury. $\mathrm{Br} \mathrm{J}$ Surg. 1993;80(8):988-91.

9. Jones JJ, Jeffreys RV. Relative risk of alternative admission policies for patients with head injuries. Lancet. 1981;2(8251):850-3.

10. Lee ST, Liu TN, Wong CW, Yeh YS, Tzaan WC. Relative risk of deterioration after mild closed head injury. Acta Neurochir (Wien). 1995;135(3-4):136-40.

11. Nee PA, Hadfield JM, Yates DW, Faragher EB. Significance of vomiting after head injury. J Neurol Neurosurg Psychiatry. 1999;66(4):470-3.

12. Ando S, Otani M, Moritake K. Clinical analysis of posttraumatic vomiting. Acta Neurochir (Wien). 1992;119(14):97-100.

13. Mild Traumatic Brain Injury Committee of the Head Injury Interdisciplinary Special Interest Group of the American Congress of Rehabilitation Medicine. J Head Trauma Rehabil. 1993;8:86-7.

14. Graham CA, O'Toole SJ, Haddock G. Neonatal head injuries. J Accid Emerg Med. 2000;17(5):334-6.

15. Macedo KC. Características clínicas e epidemiológicas de crianças e adolescentes com traumatismo cranioencefálico leve e análise de fatores associados à fratura de crânio e lesão intracraniana [dissertação]. Belo Horizonte: Universidade Federal de Minas Gerais; 2006.

16. Diament A, Cypel S. Neurologia infantil. $3^{\mathrm{a}}$ ed. São Paulo: Atheneu; 1996.
17. Gennarelli TA. Mechanisms and pathophysiology of cerebral concussion. J Head Trauma Rehabil. 1986;1:23-9.

18. Shane SA, Fuchs SM. Skull fractures in infants and predictors of associated intracranial injury. Pediatr Emerg Care. 1997;13(3):198-203

19. Stiell IG, Clement CM, Rowe BH, Schull MJ, Brison R, Cass D, et al. Comparison of the Canadian CT Head Rule and the New Orleans Criteria in patients with minor head injury. JAMA. 2005;294(12):1511-8.

20. Thompson RK, Malina S. Dynamic axial brain-stem distortion as a mechanism explaining the cardiorespiratory changes in increased intracranial pressure. J Neurosurg. 1959;16:664-75.

21. Dunning J, Batchelor J, Stratford-Smith P, Teece S, Browne $\mathrm{J}$, Sharpin $\mathrm{C}$, et al. A meta-analysis of variables that predict significant intracranial injury in minor head trauma. Arch Dis Child. 2004;89(7):653-9.

22. Da Dalt L, Andreola B, Facchin P, Gregolin M, Vianello A, Battistella PA. Characteristics of children with vomiting after minor head trauma: a case-control study. J Pediatr. 2007;150(3):274-8.

23. Jan MM, Camfield PR, Gordon K, Camfield CS. Vomiting after mild head injury is related to migraine. $J$ Pediatr. 1997;130(1):134-7.

24. Lückhoff $\mathrm{C}$, Starr M. Minor head injuries in children - an approach to management. Aust Fam Physician. 2010;39(5):284-7.

25. Haydel MJ, Preston CA, Mills TJ, Luber S, Blaudeau E, DeBlieux PM. Indications for computed tomography in patients with minor head injury. $\mathrm{N}$ Engl $\mathrm{J}$ Med. 2000;343(2):100-5.

26. Oman JA, Cooper RJ, Holmes JF, Viccellio P, Nyce A, Ross $\mathrm{SE}$, et al. Performance of a decision rule to predict need for computed tomography among children with blunt head trauma. Pediatrics. 2006;117(2):e238-46.

27. Chan $\mathrm{KH}$, Yue $\mathrm{CP}$, Mann KS. The risk of intracranial complications in pediatric head injury. Results of multivariate analysis. Childs Nerv Syst. 1990;6(1):27-9.

28. Hugenholtz H, Izukawa D, Shear P, Li M, Ventureyra EC. Vomiting in children following head injury. Childs Nerv Syst. 1987;3(5):266-70.

29. Jeret JS, Mandell M, Anziska B, Lipitz M, Vilceus AP, Ware $\mathrm{JA}$, et al. Clinical predictors of abnormality disclosed by computed tomography after mild head trauma. Neurosurgery. 1993;32(1):9-15.

30. Miller EC, Holmes JF, Derlet RW. Utilizing clinical factors to reduce head CT scan ordering for minor head trauma patients. J Emerg Med. 1997;15(4):453-7.

31. Vilke GM, Chan TC, Guss DA. Use of a complete neurological examination to screen for significant intracranial abnormalities in minor head injury. Am J Emerg Med. 2000;18(2):159-63.

\section{Endereco para correspondência}

Carlos Umberto Pereira

Av. Augusto Maynard, 245/404, Bairro São José

49015-380 - Aracaju, SE, Brasil

E-mail: umberto@infonet.com.br 